\title{
Problemas de la Política pública educativa regional
}

\author{
Juan D. Ruiz \\ (Universidad Nacional de la \\ Patagonia Austral. Argentina)
}

\section{Resumen}

Las políticas públicas educativas de los años noventa, centradas en temas como la equidad, la calidad, la autonomía y otros conceptos estelares no tuvieron el impacto necesario al momento de enfrentar el modo de pensar cómo articular la educación con el mundo del trabajo. El caso de la provincia de Santa Cruz no fue una excepción en este sentido, lo que dejó en manos de las determinaciones del mercado de trabajo las orientaciones y estímulos acerca de qué era lo necesario para definir prioridades y criterios en este campo especial del comportamiento del sistema educativo. Por efectos de los procesos desreguladores, de privatización de las Empresas estatales (YPF, YCF, Gas del Estado) y mercadocéntricas sucedidos en la década de los noventa se produjo un recambio e innovación tecnológicos importantes en diferentes sectores de la actividad económica provincial. Las evidencias empíricas originadas en investigaciones sobre esta cuestión indican que la formación básica y específica que animaba los ejes de la reforma se "adaptaron" a las lógicas que emergían de tan complejo proceso.

\section{Palabras clave}

Reforma educativa - Privatización - Formación básica y específica - Lógicas estructurales - Innovaciones tecnológicas - Reformas organizativas.

\section{Summary}

The public education policies of the nineties, focusing on themes such as equity, quality, independence and other stellar concepts did not have the necessary impact when facing the way of thinking on how to articulate the education with the working world. The case of the Province of Santa Cruz was not an exception in this sense that deferred to the determinations of the labor market guidance and encouragement about what was necessary to define priorities and criteria in this particular field of educational system behavior. For purposes of deregulatory processes, privatization of state enterprises (YPF, YCF, Gas del Estado) and centralized markets occurred in 
the nineties there was a replacement and major technological innovation in different sectors of the provincial economy. The empirical evidence stemming from research on this issue indicates that the basic and specific training that encouraged the thrust of the reform is "adapted" to the logic that emerged from this complex process.

\section{Key words}

Educational reform - Privatization - Basic and specific formation - Structural logic Technological innovations - Organizational reforms.

\section{Problemas de la Política pública educativa regional. Lógicas estructurales y educación posreforma}

El presente trabajo reconoce sus antecedentes en una serie de estudios realizados en la localidad y la provincia sobre atributos importantes del mercado de trabajo, sobre la reforma educativa y sobre la relación entre educación y trabajo en mercados regionales desregulados en la década de 1990 y en los años siguientes. Los escenarios que se re-estructuraron en esa década han modificado sustancialmente el modelo de mercado de trabajo protegido y formal que fue consecuencia del estilo de desarrollo de los años 60-70, el que se vio desarticulado y fragmentado impulsando la exclusión, la informalidad y nuevas formas de subjetivación de los sujetos (Ruiz y Salvia, 1997a, 1997e).

El referido a la reforma, como esfuerzo metódico, abordó el estado de transformación que se pretendía realizar sobre la idea de autonomía de la organización escolar y en cómo se establecía la propuesta, desde las instancias de la administración y gobierno del sistema educativo provincial, con la escuela, el modo de Dirección y el equipo docente (1). En este sentido se abordan las coordenadas de política general y educativa que emerge de la poscrisis del Estado provincial, las directrices de la reforma administrativa que impulsó el nuevo gobierno surgido en 1991 y la reforma educativa sostenida en la Ley Federal en el año 1994 a partir del Acuerdo Federal que firmó la provincia (Ruiz, 2005d).

El más reciente se inscribe en el abordaje de lo que en un Proyecto de Áreas de Vacancia (2), se denomina Saberes Socialmente Productivos y los modos histórico-regionales con que fueron generados y trasmitidos entre generaciones, su relación con entramados sociales y los procesos de subjetivación y formación de individualidades vinculados a los mercados de trabajo. En el caso del modelo de desarrollo del espacio patagónico se abordó la segunda fase del desarrollo socio-económico de la provincia después de 1940, que fuera inducido por el desarrollo del modelo de enclave petrolero, con la crisis del enclave ovino como fondo, potenciado por las ideas desarrollistas de las décadas siguientes. En ese estudio encontramos líneas y directrices de cómo el modelo de Mercado 
de Trabajo protegido que acompañó ese desarrollo, produjo condiciones favorables para la trasmisión de un conjunto altamente formalizado y calificado de Saberes del trabajo y de las subjetividades e identidades que anclaron en esa idea de ocupación territorial y desarrollo social (Ruiz, 2008e).

Creemos, asimismo, que la política educativa tradicional, entendida como elemento del proyecto de transformación global que suponía un modelo de política general definida desde lo macroestructural, entró en crisis sobre todo, después del desarrollismo instrumental a fines de la década de 1980 y la reforma estatal de inicios de los noventa. Lamentablemente el contenido, el programa, la orientación normativa y la competencia social de la reforma neoliberal de los noventa desplazaron aquel enfoque y re-formatearon la vieja escuela pública, dejando secuelas y resultados poco alentadores de esperanzas colectivas y expectativas sociales. Dada la creciente y sistemática evidencia empírica que arrojan las distintas líneas de investigación sobre este tema, no nos detendremos en profundizarlo.

En un trabajo contextualizado en líneas de investigación que abordan los problemas del mercado de trabajo, distribución de los ingresos y otros temas vinculados a este complejo campo, Danani y Grassi (2008) señalan que no se percibe en la actualidad un proyecto que haya recompuesto o haya construido otra hegemonía alternativa a la neoliberal de los noventa. El estudio afirma que es necesario reconocer una crisis de la hegemonía neoliberal antes que afirmar la crisis del neoliberalismo. Es bajo esta perspectiva que entendemos que, en los escenarios escolares emergentes de los últimos años, se cuecen nuevas subjetividades, menos interesadas por el conocimiento, más fragmentarias, menos solidarias y sometidas a procesos de desafiliación social diversos, siendo uno de los más críticos el del mundo del trabajo. Los sujetos se reconfiguran, a pesar de todo, en espacios "líquidos", de difusas maneras que apenas delimitan la incierta frontera entre inclusión y exclusión social.

Por eso se vuelve necesario plantear el tema desde lo regional en tanto, la falta de un destino común, que origina el mensaje del neoliberalismo globalizador de fines del siglo XX impacta de modo intenso en la idea de encontrar sentido a una nueva manera de pensar la escuela. Imaginar una educación obligatoria de calidad e igual para todos es, para los que queremos desplazar del centro a las lógicas de ese modelo alienante y de alabanzas a la idea de competencias, jugarnos por una trama cultural en la que se resignifique, se reterrorialice la idea de educación asociada a principios de solidaridad, compensación y de igualdad en la apropiación de los conocimientos. El desafío para las políticas educativas del estado provincial en su relación con los procesos de re-configuración del orden que desencadenó la desregulación y transformación estructural del Estado, es atender política, económica y socialmente esa trama en consolidación. Es imprescindible operar con diversas e innovadoras estrategias en el campo de la formación general y especial frente a los siguientes procesos de estructuración general que se consolidaron bajo la reforma estatal a saber: 
1. los que se originan y dan sentido al dominio de las lógicas de producción y distribución de bienes materiales y simbólicos que emergen como efectos de la hegemonía de las grandes empresas extractivas en la región y que impactan en el capital social acumulado y en los modos en que el mercado de trabajo impone lógicas instrumentales de selección de personal. Esta trama poderosa de conformación socializadora re-crea las expectativas educativas y sociales colectivas e individuales, modelando un aspecto no menor del imaginario social (petróleo, minería, gas, pesca);

2. las lógicas de reingeniería que aceleran los criterios de adaptación cualificante o adaptativa que ha puesto en marcha la transformación tecnológica y de gestión organizativa en un conjunto de empresas de distintas ramas de la actividad económica bajo el criterio de disminuir costos, elevar la productividad y mejorar la rentabilidad. Se necesita aclarar que son sustancialmente diferentes de las que se registran en la dimensión del trabajo público que exhiben un marcado retraso en las formas organizativas y las técnicas resultantes;

3. la lógica de estructuración presente en el entramado social que emerge como efecto de la aplicación generalizada de planes y programas de subsidios, promoción social, asistencialismo y contención (planes Trabajar I, II, Plan Joven, Planes Jefes y Jefas de Hogar, Programa de promoción de cooperativas, Subsidios de desempleo, Subsidios privados a trabajadores sin trabajo en convenio con Municipios, Comedores escolares, etc.);

4. las mismas lógicas inerciales de estructuración que imponen las políticas educativas actuales que, pese al bajo impacto real, se mantienen aún para transformar la escuela en base a los principios centrales de la reforma noventista: descentralización, desregulación, autonomía escolar, profesionalización docente, gestión y participación, equidad entre otras.

\section{Lo que se acepta o lo necesario}

No es objetivo de este análisis reflexivo imaginar o presentar un conjunto definido de estrategias y esquemas operativos que requiere la compleja situación actual, sino avanzar en algunas cuestiones críticas en este campo, cuestiones que pueden pensarse como sustantivas al desarrollo local. Las evidencias con que contamos parecen indicar que, determinadas políticas administrativas que desarrolla el estado provincial, exhiben escasa preocupación y direccionalidad estratégica, por tanto, probabilidades de influir, en el mediano y largo plazo, en el diseño y en los modos de organización que adoptan sectores productivos y que, debe reconocerse, impactan en los perfiles y el volumen de la demanda de trabajo. De esta manera se puede admitir razonablemente que, tales elementos, se sobreponen a las coordenadas educacionales a la hora de pensar en la formación de sujetos que pretenden integrarse en ámbitos 
laborales reproducidos o transformados por las empresas. En tal sentido cabe preguntarse qué finalidades y al servicio de quiénes se propone reorganizar e instrumentar el aparato de socialización y de preparación profesional formal que depende de las políticas públicas educativas frente a las lógicas ya citadas. El tema merece cuanto menos, una preocupación colectiva para construir la evidencia empírica que explicite algunas cuestiones en relación a este tipo de problemas (3).

No basta con que sólo se piense y exprese que esa Formación es necesaria para una vida social y productiva con el sentido incluyente indispensable para mejorar las condiciones de una sociedad democrática que prioriza la calidad de vida, la igualdad, la participación social. El discurso oficial sólo parece admitir cierta incapacidad o debilidad para influir sobre la dirección y las finalidades operadas desde el segmento de sectores más o menos dinámicos y en crecimiento del aparato económico a los que parece "adaptarse" pasivamente. Sectores responsables, junto con el del trabajo público con una importancia no reconocida, del rediseño, comportamiento y perfil de la demanda de trabajo en los últimos años. Existen, en este tema crítico para los trabajadores jóvenes, dos claras tendencias que hemos observado como elementos estructurales del modo en que, sectores de la economía, han logrado re-formatear el modelo organizativo y los diseños del trabajo que les permitieron enfrentar las nuevas condiciones del mercado (Ruiz, 2004b).

Ellas son un aumento de las certificaciones escolares en la fuerza de trabajo y un diseño de trabajo que posee aires de familia con el viejo esquema Taylor-fordista de división de tareas y asignación de atribuciones en la organización (Zárate y Artesi, 2004). Asociado a este modo de resolver los mayores niveles de incertidumbre y competitividad que introducen los principios desregulatorios y privatizadores en la actividad económica, aquellos mayores niveles de escolaridad no se traducen en reconocimientos salariales que mejoren las expectativas de la fuerza de trabajo y del conjunto social hacia el papel del conocimiento como fuente de oportunidades individuales y familiares. Sólo una minoría de los asalariados ocupados, los de alta calificación, se benefician con los logros del crecimiento, la participación profesional y técnica y la distribución de la riqueza que produce la sociedad. Las tramas productivas que han surgido desde los años noventa no vierten su mayor potencial en dirección a la idea de un desarrollo local fuertemente integrado con las necesidades del estado y la sociedad.

El proceso induce/alimenta la aptitud de los sectores empleadores por la que, en una fase posdesreguladora el excedente de mano de obra, con mayor calificación o escolaridad, crea las condiciones para el aumento de la competencia por un menor volumen de trabajo formal que favorece el credencialismo. De manera que, por ejemplo, los que poseen esos mayores niveles de escolaridad terminan por desplazar a los menos calificados de puestos de trabajo de más 
baja calificación y fortalece la tendencia para la ocupación de ciertos empleos antes realizados por los menos educados (Beccaria y Mauricio, 2008). Una mayoría (60-70\%) de los jóvenes con mayor escolaridad que, muchas veces temporalmente, acceden a una ocupación lo hacen en posiciones de baja calificación (operativa y sin calificación) y en situación informal. Se incluyen en tramas productivas que subutilizan la fuerza de trabajo y el capital social (Ruiz, 2004b, 2004c). Al tiempo, la caída de los ingresos de los deciles más bajos de la escala distributiva se acompaña de una mayor desigualdad en la apropiación de los conocimientos que ofrecen los programas escolares. Cabe reconocer que la perspectiva de un vasto conjunto de Programas y Cursos de recalificación o capacitación de trabajadores e incluso las perspectivas educativas de programas de enseñanza ha instalado, como visión dominante, la cuestión de la formación en competencias.

\section{¿Son las Competencias una alternativa innovadora?}

Una de las virtudes que han mostrado, en las últimas décadas, ciertos modelos o enfoques en campos diferentes pero vinculados, tales como el referido al trabajo y a la educación, es la capacidad para reformatear sus principios y dispositivos con el fin, más o menos sutil o encubierto, de presentarse como novedades o innovaciones frente a las críticas que resaltaban sus limitaciones sociales, laborales y educativas. Ese atributo ha sido el que ha posicionado el tema de las competencias como un germen de creatividad desafiliado de sus vínculos históricos con la extensa progenie que lo enmarca y califica su continuidad (4). Nosotros creemos que el modelo de las competencias representa un desarrollo, creativa e interesadamente manipulado, de lo que significaba (en medida importante) el automatismo humano asociado al modelo taylorista de diseño de trabajo. Como sabemos esta escuela, que se pretendía sostenida en el cientificismo, proponía, centralmente, un "ser productivo" que sólo debía ocuparse de sí mismo como factor de producción, como empresario de su propia fuerza de trabajo (mercancía). Representaba el autómata que puede perder todo si entra en contacto con el entramado externo que, en definitiva, lo significa y lo constituye como sujeto.

A modo de metáfora podemos decir que el sistema de las competencias es al trabajador lo que el sistema dinámico era respecto al modelo clásico de la ciencia en el que todo era determinista y reversible, fragmentable y articulable. El hombre competente del modelo neoliberal presenta un parecido de familia, cierta profunda afinidad con el autómata de la máquina simple en tanto puede reformarse desde cualquier estado y reformatearse con nuevas competencias (a modo de instrumento reversible) y sólo porque el conocimiento de las condiciones de competitividad permiten conocer cuando deja de ser competente el sujeto del trabajo (determinismo) (5). Así el trabajador, sus saberes, capacidades, habilidades y creencias, histórica y socialmente engendradas, pasan a 
ser completamente manipulables para quien sabe interpretar los estados del sistema y sus problemas de equilibrio.

El modelo tiende a excluir la posibilidad de trayectorias diferentes o de las bifurcaciones que, como atributos de las subjetividades sociales y las cambiantes condiciones contextuales, sólo hace necesario imaginar diferentes fases hacia la idea de competencia global. En este esquema pasan a considerarse como ruidos y perturbaciones (que hay que neutralizar como en el caso de los tiempos muertos en el trabajo) aquellas situaciones que disminuyen o erosionan el nivel de competencia de cada unidad productiva en tanto impactan la productividad del sistema y reducen la rentabilidad. El principio básico que alimenta este enfoque es el de la mayor eficiencia para el mejor rendimiento, que siempre constituyó la matriz de las ideas taylorianas respecto al tiempo y movimiento.

Podemos aceptar que este modelo tecnológico y de estrategia de entrenamiento en contextos artificiales (enfoque de las competencias) propone el aprendizaje de "competencias descontextualizadas" que se diferencia, inevitablemente, de una perspectiva en la que el aprendizaje del saber y el saber hacer representan una forma de innovar el núcleo básico de SSP (6). Conjunto de saberes que representan la inteligencia social acumulada por una sociedad o sectores sociales en su interacción y transformación con el medio/contexto que se resiste a un tipo de instrumentalización que, por constituirse desde un exterior racionalizado, conlleva impensables consecuencias, como lo muestra la historia. En este esquema racionalista (medio/fin) de la tecnología, el trabajador competente es el que conecta distintos cursos de acción alternativos (por ejemplo una técnica, un dispositivo, una herramienta) con estados reales (como la fase de producción, ensamblaje, control de calidad, etc.) en dirección a optimizar la productividad (elevando la rentabilidad).

Como afirmamos en el Informe Final de PAV sobre los SSP antes mencionado, en el modelo de las competencias "El componente educativo del trabajo se diseña de manera tal que tiende a reducir el sentido mismo de este último a una serie de operaciones que corresponden a las acciones sucesivas que debe llevar a cabo un trabajador en un puesto de trabajo. Es lo que llamaron 'competencias'. La capacitación para la adquisición de competencias pasa a sustituir, en los diseños curriculares, tanto a los contenidos denominados 'clásicos', como a los que surgen del medio cultural y social regional, nacional y local, y también los que son 'útiles' o 'prácticos' (como diría el antiguo pero aún vigente discurso de la 'Escuela Activa') para la vida social, para la formación política del ciudadano y para su relación con la naturaleza, con intenciones democráticas. Este último enunciado debe subrayarse porque la pedagogía neoliberal tiene intenciones de formar ciudadanos sin intenciones democráticas, con objetivos selectivos, para alimentar un orden meritocrático que sirve al capitalismo salvaje". 


\section{Política educativa y pensamiento administrativo}

La baja disposición que aún caracteriza el comportamiento del aparato estatal provincial para reestructurar y profesionalizar sus esquemas de orden administrativo y regulador de las actividades económicas, las evidencias del fracaso de la reforma en educación y, los principios y procedimientos que reorganizan el mundo productivo son factores que "auspician" el predominio de las lógicas del mundo económico por sobre las educativas. La capacidad para ejercer influencia en las tendencias, procesos y en la formación de sujetos con el tipo de conocimientos, saberes y técnicas generales y específicas, que interpele críticamente aquellas tendencias economicistas, aún carece del peso específico necesario para impactar en los esquemas y la praxis de las organizaciones económicas. Más allá de los acuerdos que el Estado pueda alcanzar con áreas de la producción -áreas en las que suele dominar el interés de tales sectores- la política educativa debe avanzar hacia la definición de finalidades iniciales más protectoras y formadoras de los sujetos implicados en estos procesos. Debe enmarcarse en esquemas de análisis que den cuenta satisfactoriamente de la posición subordinada que el nuevo régimen de acumulación regional impone a los programas de política estatal, en particular la educativa y al papel aquiescente que caracteriza al modelo político dominante en esta trama.

La descentralización, operada por los procesos reestructuradores, en el marco del "modo autoritario y centralizado" con que se implementó en la provincia a inicios de 1992 (Ruiz y otros, 2005d) anula las diferencias que aquella idea proponía reconocer, alienta al insularismo cultural-educativo y la diferenciación insolidaria que demora la afluencia de una diversidad de proyectos en lo local o regional en torno a la educación. El modo en que se operan aquellas ideas de transferencia de la autoridad quedó a la interpretación y operacionalización de unidades escolares poco dotadas en infraestructura tecnológica, profesional, de gestión y de apoyo técnico-pedagógico. Tal situación tiende a potenciar el modelo clásico de reproducción escolar de las desigualdades sociales que había sido uno de los problemas más graves sobre los que se asentaron las propuestas e ideas de cambio y mejora de las escuelas. La situación merece reconocerse para abrir posibilidades acerca de mejores estrategias en la atribución de autonomía real a las unidades escolares que se proponían como base de la atención a la diversidad.

Tanto como propender la mejora constante de los aprendizajes diferenciales e incrementales que las escuelas y los docentes deben asegurar, es necesario trabajar sobre la superación de los cada vez más desiguales puntos de partida de las generaciones escolares que inciden, de modo inevitable, en la mejora de las condiciones de educabilidad de esos sujetos. Ya señalamos que la tendencia inflacionaria y la crisis recesiva de los últimos dos años impacta en este escenario y, es bastante probable, esperar mayor desigualdad en este punto sustantivo de la reparación cognitiva de los sujetos escolares para incorporarlos 
a la cultura escolar. La distribución regresiva del ingreso provincial, a pesar de los discursos sobre el crecimiento de la economía provincial, tiene una influencia decisiva en los hogares de menores salarios y aumenta las posibilidades de cristalizar las pobres disposiciones de esos hogares frente al consumo de bienes simbólicos. Los niños provenientes de estos sectores presentan una disposición intelectual menos afinada respecto al mensaje lingüístico y pedagógico de la escuela.

Así al no percibir críticamente y tomar debidamente en cuenta aquellas lógicas que contribuyen a cristalizar las condiciones de un nuevo entramado societal que contribuye a distribuir y utilizar desigualmente los recursos científicos (conocimiento), tecnológicos y culturales, se debilitan las posibilidades de trabajar en pos de una mejor distribución de la riqueza, del conocimiento y otros bienes básicos que aseguren la cohesión social en términos de crecimiento y desarrollo local. La impronta conservadora que se impuso a lo largo de la década de los noventa y aún persiste con sus secuelas de individualismo y fragmentación requiere de un pensamiento educativo esperanzador que encuentre los núcleos o nudos gordianos de los actuales entramados que generan aquellas tendencias que alimentan la desigualdad de oportunidades. ¿Hay acaso o pueden observarse en el actual discurso oficial sobre el cambio de la escuela indicadores suficientes de un idealismo utópico que permita que no se defrauden las esperanzas de justicia social que la escuela puede ofrecer a las masas populares hoy más pauperizadas? ¿O los fines de la educación presentan una sintonía con los fines de la distribución desigual de poder y por tanto del acaparamiento de las oportunidades sociales, culturales y educativas en sectores privilegiados de la sociedad?

El incremento de productividad observado en los procesos productivos y de servicios en esta localidad se logró con la incorporación de máquinas o aparatos que incorporan innovaciones grandes o pequeñas. Como señalan los especialistas la innovación tecnológica suele ser, en mayor medida, un proceso de "difusión" seguido de una "imitación creativa" que a veces, comienza a ampliar la función creativa y se vuelve innovación completa o en sentido total. El aumento de la demanda real (mayor consumo de bienes) que supera el nivel de la productividad media de la economía aumenta la demanda de empleo por incremento de la del Trabajo. Pero si el aumento de los salarios se coloca por encima de los precios de las máquinas, genera un estímulo que favorece la introducción de las innovaciones que se incorporan en máquinas y que ahorran trabajo al tiempo que se incrementa la productividad (por hora/ hombre). En estos casos para capturar mano de obra calificada los empresarios generan un movimiento de crecimiento de los salarios (7) que presiona al incremento de la productividad y reduce el coeficiente de trabajo (ahorro). De manera que, históricamente, en los procesos de casi pleno empleo el costo del trabajo aumenta sistemáticamente y las innovaciones ahorradoras de trabajo son más frecuentes. 


\section{Consideraciones finales}

Qué formación para el trabajo pensar al analizar la demanda y las calificaciones que se configuran en este tipo de mercado de trabajo es una instancia compleja y crítica que requiere información relevante y relativamente actualizada dada la dinámica que imprimen los procesos en escenarios cambiantes e inestables. A partir de trabajos anteriores (Ruiz, 2004b; Ruiz y Muñoz, 2005c), podemos indicar que el panorama de desarrollo local/regional, permite reconocer que un tipo de dinámica evolutiva del sector se presenta apropiado para pensar ciertas respuestas a temas claves detectados en los Análisis de Coyuntura Laboral y Económica (2do. Semestre 1994 - 1er. Semestre 1995) presentado al MTySS a partir del Convenio de este organismo con la UNPA y que fueran dirigidos por el A. Salvia y los equipos de investigación que abordaron ese objeto de estudio.

En un contexto económico de mayor crecimiento, desde la recuperación del 2002 y con la actividad petrolera con ritmos sostenidos de actividad junto al crecimiento del conjunto de obras públicas provinciales, el sector Secundario ha incrementado su participación relativa en la estructura económica. Siendo éste un dato importante también lo es el hecho de que un conjunto de empresas del sector parecen haber mejorado/innovado relativamente sus modos de organización productiva. Sin embargo, no aparecían evidencias fuertes de haber iniciado un camino hacia la mayor eficiencia en las formas de gerenciamiento más apropiadas a los nuevos escenarios de competencia y calidad.

Al impulso inicial hacia esta forma de desarrollo, basado en mayores y mejores niveles de productividad y márgenes de rentabilidad, le faltaron la creación de líneas crediticias que hacen posible un financiamiento dirigido al aumento de la demanda real en términos de calidad y que indujera un aumento de la demanda de trabajos más calificados. Este panorama actual de mejoramiento no impacta en el aumento de diferentes líneas/programas de capacitación relativamente permanentes sobre la Formación/Calificación del personal Profesional-Técnico -como aquellos que desempeñan tareas de Operación, Administración, Asistencia técnica, Apoyo a incrementar diseños o de re-ingeniería de procesos, Rediseño de líneas de comunicación en y entre equipos y otras- como lo observamos en encuestas a informantes calificados recientemente. Estas líneas que son una necesidad empresarial para la selección y calificación de los trabajadores de empresas locales deberían imitar aquéllas que, en aquel Informe, ya había iniciado el grupo de unidades de Servicios varios vinculados a la actividad petrolera, actividades que antes se cumplían al interior de la empresa estatal.

En ese amplio estudio descriptivo-interpretativo señalábamos que la demanda y el perfil de trabajador en las Empresas analizadas se orientaban hacia empleados del sector de Supervisión y Mantenimiento de instalaciones y a Operarios altamente calificados (soldadores de tuberías de alta presión, 
mecánico de motores y turbinas, electro-instrumentalistas y otros). A este tipo de trabajadores se les ofrecía un programa diferencial de capacitación, dada la reciente incorporación de Tecnología y Modos de producción requeridos para dar respuestas a las mayores exigencias de las Empresas Petroleras que exigían mayor Calidad en los servicios que subcontrataban. Paralelamente, se generaban mayores niveles de incertidumbre y de competitividad en esta rama de la economía para ocupar y mantener los nichos de mercado altamente rentables que dependen de la producción energética en la provincia.

Sostenemos que los datos sistematizados en esos estudios y otros más actuales realizados en torno al comportamiento del Mercado, el Empleo y las Calificaciones, así como de otros colegas de la región, muestran la presencia de dos procesos o tendencias que merecen la atención profunda y preocupada desde las políticas educativas. Por un lado se observa que la estructura del empleo en la economía provincial, tanto en el sector privado como público, presenta un alto grado de trabajadores con bajo nivel de calificación (más de las $2 / 3$ partes) paralelamente a un crecimiento de los niveles de escolaridad de la fuerza de trabajo ocupada. En este último grupo de empleados se destaca en el mercado de trabajo el incremento de la participación femenina. En segundo lugar, los análisis destacan que en los últimos 10 años, los segmentos que prevalecen en el volumen de la demanda de empleo han sido los sectores que conforman el nivel Terciario: servicios en general (a las empresas, financieros, de salud, educación, doméstico, etc.), comercio, transporte y otros. Conocer con mayor detalle las tendencias que impulsan ese fenómeno así como los rasgos básicos de la formación que comparten los trabajadores semicalificados y con mayor calificación, utilizando estudios de casos considerado nodales, se convierte en una herramienta necesaria para re-definir líneas curriculares que promuevan la tarea de enseñanza en el nivel básico y especial referidas a la formación para el trabajo.

Esa información empírica señala que el aumento de la subutilización de la mano de obra y el incremento de los niveles de escolaridad (8) de la fuerza de trabajo, a pesar de la baja calidad de los empleos a los que se accede, crecen de la mano del empleo precario y no registrado informal. El fenómeno reproduce $y$, en momentos declinantes del ciclo económico, incrementa la distancia entre los ingresos inferiores y los superiores. Este contexto poco favorable para el conjunto de trabajadores, en particular jóvenes y jefes de hogar poco calificados, de las pequeñas empresas que configuran más del $80 \%$ de la estructura económica provincial, produce un efecto de desaliento individual y colectivo respecto a la educación y otros bienes simbólicos.

El fenómeno genera un efecto hacia la disminución de expectativas de capacitación (formal o informal) y empleo e impulsa a tales grupos de trabajadores a aceptar puestos de trabajo con mayores niveles de precariedad, poco calificados, inestables y, en general, no registrados. De manera que la población 
trabajadora más joven aparece sobrerepresentada en estos sectores de la ocupación. Aunque los jóvenes pueden poseer niveles de escolaridad altos, ello no suele tener la suficiente importancia en el plano del diseño de tareas en la medida en que, para los empresarios, prevalece la valoración de la antigüedad o experiencia laboral a la par que bajas expectativas en la participación del trabajador en el cambio organizacional que los mayores grados de competencia y adaptabilidad al contexto requieren.

Uno de los interrogantes que merece atención en base a lo que hemos descripto es imaginarse cuánto de aquellos rasgos que estamos observando en términos de eficiencia escolar están asociados a los procesos sociales, laborales y familiares que abordamos y en qué medida son funcionales a este cambio de la lógica económica basada en transferencia de capitales y en una regresión distributiva. Siendo coherentes con nuestra crítica acerca de aquellas posiciones que simplifican el modo de entender la articulación entre la educación y el mundo del trabajo podemos señalar lo siguiente. Al tiempo de reconocer que existe un tipo de vínculo más o menos visible entre ellos, el contexto histórico-social y económico sujeto a la desregulación reterritorializan un tipo de relaciones, atributos y direcciones que, en este caso, subordinan o desvalorizan componentes y elementos de imaginarios escolares alternativos en su vinculación a las necesidades del sistema productivo.

\section{Notas Bibliográficas}

(1) Proyecto denominado: "Política educativa en contextos posautoritarios: Entre las ilusiones de mejorar la calidad y equidad educativa y las alternativas que imponen los principios de estructuración después de la reestructuración estatal". UARG-UNPA. Acuerdo 353/03.

(2) PAV No 153 denominado: "La Enseñanza y aprendizaje de los Saberes Socialmente Productivos. Los Saberes del Trabajo". Dir. Dra. Adriana Puiggrós. ANPCyT-FONCyT. 2005-2007. (Desarrollado en forma de red con equipos de universidades públicas nacionales: UBA, UNR, UN de San Juan y UNPA).

(3) En un programa de Extensión científica y técnica organizado por la Universidad, tuvimos la oportunidad de interactuar, en 2007 , con estudiantes de carreras técnicas en la localidad y percibimos sus críticas expectativas sobre aspectos de la formación, la vinculación con el mercado de trabajo y la escasa dotación de equipamiento tecnológico en los talleres que les posibilitara un conocimiento e ingreso más apropiado a las condiciones del mundo del trabajo. La década de los noventa registró procesos de innovación tecnológica en empresas de los sectores de servicios en general y, en especial, de los Servicios a Empresas petroleras (Salvia y Ruiz, 1997; Ruiz, 2004).

(4) El lenguaje de las competencias, con acento en su forma técnica, no reconoce explícitamente la larga tradición que la enmarca, mientras pone énfasis en su 
eficacia para reconstruir las competencias del trabajador. (Dejours (1998) "El Factor humano". Buenos Aires, Lumen).

(5) Tal como lo especifica Spinoza (2008) esta escuela debe entenderse como una nueva tecnología de regulación del trabajo y de búsqueda de nuevas formas de clasificación desligadas del sistema protectorio de legislación laboral, en Novedades Educativas; Año 20, № 207, Marzo, pp.4-11.

(6) Entendemos por Saberes Socialmente Productivos (SSP) "aquellos saberes que modifican a los sujetos enseñándoles a transformar la naturaleza y la cultura... se conforman histórica y socialmente, se trata de saberes que engendran, que procrean y tienen fuerte vinculación con elaborar y fabricar", en Puiggrós, A. y Gagliano, R. (Dir.) (2004) La Fábrica del Conocimiento. Buenos Aires, Homo Sapiens.

(7) Es altamente probable que, luego de la privatización de YPF-YCF, ésta haya sido una herramienta utilizada por las empresas privadas para cooptar recursos humanos calificados y con información crítica en los modos de producción, en el nivel de reservas, en la aplicación de nuevos métodos y otros.

(8) Después de más de una década de reforma educativa se registra un incremento en índices como la repitencia, deserción y otros que terminan siendo funcionales a las orientaciones fragmentadoras y precarizantes que caracteriza la dinámica del perfil del trabajo que se impone después de la reestructuración y desregulación productiva.

\section{Referencias Bibliográficas}

- Becaria, L. (2001a) Empleo e integración social. Buenos Aires, FCE.

- Becaria, L. y Mauricio, R. (2008b) "Mercado de trabajo y distribución personal del ingreso", en Lindemboin, J. (Comp.)Trabajo, Ingresos y Políticas en Argentina. Contribuciones para pensar el siglo XXI (Cap. 2). Buenos Aires, Eudeba.

- Cohen, D. (2007) Tres lecciones sobre la sociedad posindustrial. Buenos Aires, Katz.

- Danani, C. y Grassi, E. (2008) "Ni error, ni omisión. El papel de la política de Estado en la producción de las condiciones de vida y de trabajo. El caso del Sistema provisional en la Argentina (1993-2008)", en Lindemboin, J. (Comp.) ob.cit. (Cap. 6, pp. 259-298).

- Dejours, C. (1998) El Factor humano. Buenos Aires, Humanitas.

- Garcés, L. (Comp.) (2007) ¿De la escuela al trabajo? Buenos Aires, del Signo.

- Puiggrós, A. y Gagliano, R. (2004) La Fábrica del conocimiento. Rosario, Homo Sapiens.

- Ruiz, J. y Salvia, A. (1999a) "La reestructuración económica y el mercado de trabajo en Río Gallegos", en La Patagonia privatizada. Sectores que ganan, sociedades que pierden. Buenos Aires, La Colmena, pp. 171-184.

- Ruiz, J. (2004b) "Modelo de empresa y rediseño del trabajo. ¿Una dialéctica de dominación y desigualdad?", en Zárate, R. y Artesi, L.: Conocimiento, pe- 
riferia y desarrollo. Los nuevos escenarios de la Patagonia Austral. Buenos Aires, Biblos.

- Ruiz, J. y Muñoz, N. (2005c) "Perfil de la fuerza de trabajo y formación escolar. Contextos, tendencias y puntos críticos de la relación educación-trabajo", en Observatorio Patagónico. Año 5, № 6. pp. 27-32. Con referata. UACOUNPA.

- Ruiz, J. y otros (2005d) "Autonomía educativa bajo nuevos principios de estructuración provincial en los 90", en Revista Espacios Nueva Serie. Dossier "Historia, Política y Educación". Año IV, № 4, Diciembre, pp. 150-169. Con referata. UNPA.

- Ruiz, J. (Coord.) (2008e) Petróleo y Región austral Saberes del trabajo y Educación Técnica. Buenos Aires, Dunken. 